\title{
Modelling and Clinical Trials in Paediatrics
}

\author{
Philippe Jacqmin, ${ }^{1}$ Nathalie Labouret, ${ }^{2}$ François Gueyffier ${ }^{3}$ and the Participants in Round Table \\ No. 6, Giens XX*
}

1 Société Exprimo, Beerse, Belgium

2 Agence française de sécurité sanitaire des produits de santé (Afssaps), Saint-Denis, France

3 Centre d'Investigation Clinique, Lyon, France

Abstract

Clinical trials are more difficult to conduct in children, but they are even more necessary than in adults their scarcity is an ethical scandal. Mathematical models can be built that can describe both the disease process and the mechanism of action of drugs. These models can then be used to simulate the outcome of clinical trials. Inspection of the simulated results then facilitates optimisation of the trial design and proposed methods of analysis. Validation is a crucial issue for the good practice of modelling and simulation. The participants of Round Table No. 6 recommend: (i) that modelling be systematically employed; (ii) that all the required professional personnel be involved, at all phases; (iii) that all data needed are made accessible; (iv) that clinicians be trained; (v) that specialists develop training tool kits; and (vi) that universities provide appropriate training.

Keywords: modelling, simulation, clinical trials, paediatric medicine

\begin{abstract}
Modelling and simulation (M\&S) is a methodology that can be used to describe, predict and optimise the results of as yet unconducted clinical trials during all stages of drug development. The organising committee of the Giens Round Tables felt that more consideration could be given by clinical pharmacology to this topic. In 2003, a Round Table was organised where the fundamentals of M\&S were reviewed. In 2004, it was discussed as to how M\&S could successfully be applied in paediatrics. This document summarises the Round Table discussion "Modelling and Clinical Trials in Paediatrics" held during the Giens 2004 meeting. During the 3-day discussion, the regulatory, industrial and practitioner views on the need for, but also the difficulty of, performing clinical trials in the paediatric population were presented. Furthermore, the modelling approach in paediatrics was reviewed and illustrated with practical examples, and the modelbased clinical trial simulation technique for design optimisation was presented. Finally, aspects of the validation, guidelines, education and working environment relating to $M \& S$ were debated.
\end{abstract}

\section{Regulatory, Industrial and Practitioner Views on the Need for Clinical Trials in Paediatrics}

Nowadays, care in paediatrics is still characterised by the paucity of clinical pharmacology and drug development re- sources: data are scarce and globally lacking with regard to the fundamentals of clinical pharmacology, including pharmacokinetics, pharmacodynamics and safety. Many of the available drug formulations are inappropriate for use in paediatrics.

The idea that children are reduced models of adults is appealing; this follows the principle of parsimony and simplicity, which is fundamental to good practice in modelling. However, this idea is completely wrong and misleading: (i) absorption, distribution, metabolism and excretion are different in children and, moreover, are evolving throughout the maturation of the child, with dramatic changes occurring at different points in time; (ii) diseases in children are often different in nature, if not completely specific, e.g. tumours, infections, neurological troubles (Attention Deficit Disorder, epilepsy, etc.); (iii) the effects of drugs can be of different intensity, beyond the differences due to pharmacokinetic characteristics, and which can be explained mainly by the maturation processes of organs and systems (e.g. teeth, heart, brain, etc.).

US regulation has progressively influenced the research and development $(\mathrm{R} \& \mathrm{D})$ programmes of pharmaceutical companies in the paediatric field, and has included both positive encouragement to conduct paediatric research (Paediatric Exclusivity Provision in $1997,{ }^{[1]}$ which was followed by a dramatic increase of the number of clinical trials in children) and constraints (the Pae-

\footnotetext{
* For a list of participants, please see the end of the article.
} 
diatric Rule 1998,[2] the Best Pharmaceuticals for Children Act [BPCA] 2001 ${ }^{[3]}$ and the Paediatric Research Equity Act 2003 ${ }^{[4]}$ ).

In Europe, following different lobbying activities that have included previous Giens Round Tables, the marketing of drugs for rare diseases has been encouraged, e.g. by less demanding submission files, quicker responses, and the extension of market protection. New developments, due for adoption in 2006, include information on the use of drugs in paediatrics, needs-centred evaluation, request examination by an ad hoc committee of the EMEA (European Agency for the Evaluation of Medicinal Products), scientific support and a guideline for drug development in children. ${ }^{[5]}$

\section{Difficulties and Value of Clinical Trials in Paediatrics}

Clinical trials are difficult in children because of the following: (i) specific regulations that are justified to protect such fragile individuals: there is no possibility that clinical trials can be performed in healthy children using the same approaches as in healthy adults; (ii) the populations are generally small when grouped according to age; (iii) limitations in the number and size of blood samples allowed; and (iv) the special emphasis given to the prevention of pain or anxiety.

However difficult, clinical trials are even more crucial now because of the wide prevalence of prescription drugs that have not been approved for use in children (56\% of hospital prescriptions), ${ }^{[6]}$ the lack of appropriate drug formulations (between $50 \%$ and $90 \%$ of drugs prescribed for children in Europe), ${ }^{[7]}$ and the absence of specific pharmacokinetic, efficacy and safety data. There are approximately 100 million children currently in the EU. Usually, the paediatrician has the choice of either prescribing off label and taking the risk of deleterious and potentially lifethreatening effects or a lack of efficacy, or of not prescribing potentially useful drugs for which benefit has been shown only in adults.

Therapeutic catastrophes that followed the use of sulfanilamide in the 1930s and of thalidomide in the $1960 \mathrm{~s},{ }^{[8]}$ as well as numerous other examples of serious adverse effects observed in children and not in adults illustrate the particular susceptibility of children to adverse drug effects. ${ }^{[9]}$ This risk is greater in children because of the lack of specific evaluation, ${ }^{[10]}$ inappropriate use by parents and reconditioning of pharmaceutical forms developed for adults.

A meta-analysis of 17 observational studies of hospitalised children estimated the rate of adverse events at 9.5\% (CI 6.8, 12.2) with $12.3 \%(8.4,16.2)$ being serious. ${ }^{[11]}$ Moreover, $40 \%$ of the adverse effects that caused hospitalisation were life threaten- ing. In US each year, 100000 adult deaths are attributable to drugs. [12]

- In contrast to the usual thinking, there is no rationale for differences between children and adults with regard to an ethical position. Randomisation is as licit in children as in adults when conditions of equipoise are met, and the control of confusion factors requires an identical use of placebo. It is unethical not to perform clinical trials in children.

- Off-label prescribing, which is the most common situation, can be considered to be undeclared and underpowered experiments.

\section{Population Modelling in Paediatrics}

M\&S projects start with gathering the available data/information and building a model to describe it. In most cases, a population model is developed, ${ }^{[13]}$ which usually consists of the following: ${ }^{[14]}$

- A structural submodel that represents the relationships identified in the observations made in studies by mathematical equations. This submodel can be descriptive or explanatory. An example of a descriptive model is a logistic regression model that depicts a binary outcome (e.g., yes-no successful effect). An example of a semi-mechanistic model is the maturation of liver enzymes measured in vitro $\left(\mathrm{V}_{\max }\right.$ [maximal enzymatic rate] and $\mathrm{K}_{\mathrm{M}}$ [Michaelis-Menten constant]), combined with plasma protein binding and hepatic blood flow information, and which can describe the hepatic clearance of a drug in young infants. ${ }^{[15,16]}$ Explanatory models try to introduce the causality of the observation. They are also called (semi-)mechanistic models. A (semi-)mechanistic model may allow some extrapolations, whereas a descriptive model in most cases will not. In the structural submodel, observations are qualitatively and/or quantitatively expressed as mean population parameters. Pharmacokinetic (e.g. a oneor two-compartment model with first order absorption), pharmacodynamic (e.g. sigmoid $\mathrm{E}_{\max }$ [maximal effect] model $)^{[17]}$ and disease progression (e.g. viral dynamics) $)^{[18]}$ models are examples of structural submodels.

- A statistical submodel that describes the parameter variability in the population such as the interindividual, intra-individual and inter-occasion variability. This submodel also usually provides quantitative information about the unexplained residual variability (residual error) and the uncertainty on the structural and random parameter estimates. Normal or log-normal distribution of the parameters in the population, additive or proportional residual error and vari- 
ance-covariance matrix of model parameter estimates are examples of statistical submodels.

- A covariate submodel that describes the relationship between population parameters and demographic and/or disease characteristics such as weight, age, gender or score at baseline. ${ }^{[19]}$ The Cockroft-Gault equation for renal clearance and the viral load at baseline for disease progression in hepatitis $\mathrm{C}$ are examples of covariate submodels.

In population modelling, model parameters are usually estimated by mixed-effects analysis. This analysis method allows the simultaneous estimation of the structural, statistical and covariate submodel parameters. This has the property of maintaining the correlations between the components of the model and thus permit apposite model-based simulations.

\section{Modelling Using Prior Knowledge}

Modelling allows the possibility of using prior knowledge. In most cases, prior information can help in the analysis of the new data. Structural, statistical and covariate prior information relevant to a drug can be implemented in a model. The advantage of this is that the information does not need to be generated once again in the planned study. For example, for a pharmacokinetic study in children, prior information can consist of the following: (i) the structural pharmacokinetic model developed in adults; (ii) the covariate model describing the relationship between the liver and/or renal clearance with bodyweight or their maturation processes with age; and (iii) the statistical model describing the interindividual variability in the parameters and the expected residual error of the observations in the investigated population. This prior information can come from previous studies performed with the drug itself or with compounds that have similar pharmacokinetic, pharmacodynamic and/or therapeutic characteristics. It can also come from published studies on, for example, the maturation and pathophysiology of the systems involved. Whatever the prior information is used to help with (data analysis or optimisation of study design), it will usually contribute to a better identification of the risk factors and the decrease of uncertainty in predictions or decisions. Depending on the degree of relevancy of the prior information for the compound being investigated and the objectives pursued, the planned study can be designed as an explanatory study or as a confirmatory study. ${ }^{[20]}$ The use of prior knowledge is of strategic importance in paediatric studies, since the number of patients included in a study and the number of observations that can be taken are generally limited.

The use of the prior knowledge on the maturation processes of the renal clearance in young infants has been illustrated for the study design optimisation of an anti-viral drug. ${ }^{[21]}$ This leads to the recommendation of sampling times depending on the postconceptional age. Information sharing/bridging concepts between adults and children have also been illustrated for the population pharmacokinetic model building of cyclosporine in a paediatric population. In this example, precise estimation of some model parameters and covariate effects in children has been made possible thanks to the adult data.

In many cases, the use of prior knowledge from a different experiment (e.g. using data from a clinical trial in adults to extrapolate to children) carries a significant risk, which is the propagation of error from one model (with observed data) to another (without the same observations). Although such use can be justified within an identical population, objectives and design, this is not in general to be recommended outside of the context of the origin of the data, and should always be viewed with great caution.

\section{Model Qualification}

A model is a simplified view of reality and as such cannot be validated. However, a model can be qualified for specific objectives. In estimation mode, a model can be evaluated for the goodness of fit, the precision of the parameter estimates, the distributions of the individual parameters and residual error, and the adequacy of the covariate effects. In simulation mode, a model can be qualified for its predictive performances (interpolation and/or extrapolation). The metrics used in model evaluation are usually of direct clinical interest and model independent. For example, for pharmacokinetic models, the metrics can be $\mathrm{C}_{\max }$ (maximum concentration), $\mathrm{C}_{\min }$ (minimum concentration), time above a threshold concentration or AUC $\tau$ (area under curve $\tau$ ); for a disease model, the metrics can be the primary and/or secondary endpoints of a clinical efficacy trial. Qualification of the predictive performance of a model can be obtained with data used for the model building (e.g., after partition of the data in two sets, one for building and the other for qualification, or applying resampling procedures such as bootstrapping), with historical data and/or with new data. Ideally, a model should satisfy both the modelling and simulation evaluations before it can be used in decisions as to dose selection, development of dose adaptation rules or clinical trial design optimisation.

\section{Clinical Trial Simulations}

Simulations can be performed once a validated model is available. In addition to the characteristics of the model, there are numerous aspects of a clinical trial that can have an impact on the study outcome. For example, the design itself (e.g. parallel, crossover, sequential, adaptive), the number and characteristics 
of patients, the doses and dosage schedule, the number and times of observations. In addition, it can be envisaged that a customised data analysis method/plan could be needed for each simulation scenario. The complexity of the system becomes such that it is practically impossible to get any idea of the possible outcomes without performing simulations. Analogous to flight simulators, clinical trial simulators have been developed to help trial designers in selecting the optimal design based on the possible outcomes in all practical combinations. ${ }^{[22,23]}$ This technology should be seen as a way of reducing the risk of running inconclusive studies. It can never replace the study itself. It can optimise the design and/or demonstrate its limitations. Based on the conclusions of the trial simulations, the design of a study can be modified, optimised, or in some cases the study can either be approved or cancelled.

\section{Study Design Optimisation Using Modelling and Clinical Trial Simulation}

Because of ethical and practical constraints, only limited clinical experiments in children can be performed. All precautions must be taken to ensure that each new individual observation carries the highest level of information possible. This raises the question of the optimal design in paediatric studies. M\&S can help in this specific context:

- Structural, statistical and covariate model building allows the identification of the components to which the outcome of the study is most sensitive to. Performed anticipatively with prior information, modelling is an opportunity to adapt the design of the planned study according to the relevant qualitative and quantitative information.

As examples:

- the maturation of liver metabolic enzymes or of glomerular filtration in very young infants can be modelled and used to determine their quantitative impact on the dose adaptation rule, the sampling schedule, the duration of observation and the age range being investigated;

- the modelling of neutrophil counts as a function of time in children treated with chemotherapy can lead to a pharmacodynamically based safety parameter used in the optimisation of the risk/benefit ratio in planned clinical trials;

- the already observed interindividual variability in critical parameters such as clearance, $\mathrm{E}_{\max }$ or $\mathrm{EC}_{50}$ (concentration at half $\mathrm{E}_{\max }$ ) obtained from published modelling results can be used for rough sample size estimation in a planned study;

- the residual error relating to specific practicalities can be detected and reduced by better control of these practicalities or by adapting the observations to these practicalities;
- clinical trial simulations based on a qualified model allow study design evaluation and optimisation. Study outcomes can be simulated and analysed for scenarios that combine variations in doses and dosage schedules, number and times of observations, number and characteristics of patients, parameter uncertainties and model hypotheses. This approach can be used to simultaneously fine tune study designs and analysis methods and select the best scenario for the eventual study.

However, modelling has also some limitations:

- A less positive but interesting lesson-learned example is the nonanticipated lack of systemic exposure for a nasal formulation in children. The extrapolation of adult data to children did not take into account that the nasal mucosa area relative to bodyweight is significantly smaller in children than in adults.

Some applications were discussed and illustrated with examples:

- In pharmacokinetic studies in children, the choice of the analysis methods, such as a noncompartmental analysis, non-linear-mixed effects modelling or a Bayesian analysis, ${ }^{[2,25]}$ can strongly influence the design of the study. As illustrated by examples with cisapride, very different results regarding the influence of post-natal age were obtained using either population or conventional pharmacokinetic analyses. Vice versa, practical constraints in study design, such as number of samples, times of sampling, number of subjects in each age category, can limit the choice of the analysis method. This situation was illustrated for an anti-viral drug for which the empirical Bayesian estimation method was chosen as the analysis method and the number of samples per subject and times of sampling were accordingly adapted;

- In dose-range finding trials, the performance of an adaptive trial design in selecting the correct effective dose in children can be compared with that of a sequential design or a classical predefined dose-ranging design. The apparent advantage of a continual reassessment method using the Bayesian approach in determining the minimal effective dose regimen of ibuprofen in patent ductus arteriosus in premature infants was used as an illustration. ${ }^{[26]}$ In this placebo-controlled study, the predefined success criteria (the dosage regimen giving $80 \%$ and $50 \%$ closure in neonates with a gestational age of 27-29 and <27 weeks, respectively) was obtained with the required precision after enrolment of 20 subjects;

At the end of a study, it is not unusual to observe that the main question cannot be fully answered. With $M \& S$, such a situation can be anticipated and a well informed decision be taken. ${ }^{[27]}$ The performance of a study design in its ability to meet 
predefined requirements can be quantitatively evaluated and the decision can be taken to either not perform the study or to accept its intrinsic limitations. This discussion was illustrated by an example of rectal midazolam administration for sedation of infants during cardiac catheterisation in which the active dose was demonstrated to be higher than what safety data could support and the development of the formulation was abandoned. ${ }^{[28]}$

\section{Guidelines, Education and Working Frame of Modelling and Simulation}

Recommendations for good practices of M\&S do exist, e.g. those developed by the Centre for Drug Development Science (CDDS).

The appropriate use of M\&S requires specific professional abilities. Following Population PK (pharmacokinetic), ${ }^{[29]}$ exposure-response US Food and Drug Administration (FDA) guidances, ${ }^{[30]}$ modelling should not omit validation steps, through assessing the internal coherence and the fit of the model with external data, i.e. facts or other models.

The participants in the Giens Round Table No. 6 recommend the following: (i) that modelling be systematically considered and, if adopted, that it be appropriately conducted, interpreted, validated and applied; (ii) given that $\mathrm{M} \& \mathrm{~S}$ is by nature a multidisciplinary approach, all required professional skill sets should be involved at all phases, including the technical approval process of the M\&S report; (iii) that all data required for optimal modelling be accessible, through an adequate partnership between industry, academics, regulators and health authorities; (iv) that clinicians be made systematically aware of the values and used to (if not specifically trained) interpreting the results of modelling approaches; (v) that specialists in modelling techniques develop decision trees or algorithms to help non-specialists in deciding when it is appropriate to use $M \& S$; and (vi) that universities provide appropriate training for the drug specialists in the fields of clinical trial design, clinical trial conduct, analysis of PK and PK-PD (pharmacokinetic-pharmacodynamic) and PK-PD modelling and simulation.

\section{Conclusions}

Regulatory requirements that promote clinical trials in children and lead to the best quality healthcare in this particularly fragile population are slowly evolving in the right direction. $M \& S$ techniques appear as essential tools to reach the ultimate objectives of this evolution. A systematic and appropriate use of these tools will be facilitated by:

- education and training;
- the availability of user friendly models on the web for practitioners; and

- open-access databases for small populations.

\section{Acknowledgements}

D. Armengaud (CH, Saint-Germain en Laye), I. Bost (Grenoble), A. Carpentier (LEEM, Paris), C. Caulin (Hôpital Lariboisière, Paris), C. Crépin (EMF Consulting, Aix en Provence), E.-M. Fuseau (EMF Consulting, Aix en Provence), M. Gerberg (Knoll France, Chilly-Mazarin), C. Grosskopf (Roche, Neuilly-sur-Seine), F. Gueyffier (Centre d'Investigation Clinique, Lyon), N. Hoog Labouret (Afssaps, Saint-Denis), P. Jacqmin (Sté Exprimo, Beerse, Belgique), C. Laveille (IRIS, Courbevoie), C. Le Gellec (Hôpital Bretonneau, Tours), P. Marquet (CH Dupuytren, Limoges), A. Ouslimani (DRRC, AP-HP, Paris), G. Pons (Hôpital Saint-Vincent de Paul, Paris), U. Simeoni (La Timone, Marseille), N. Simon (Faculté de Médecine, Marseille), B. Tranchand (Faculté de Médecine, Lyon), J.-M. Treluyer (Hôpital Saint-Vincent de Paul, Paris)

\section{References}

1. Section 111: Pediatric studies of drugs. Food and Drug Administration Moderization Act of 1997 [online]. Available from URL: http://www.fda.gov/cder/pediatric [Accessed 2005 Aug 8]

2. Pediatric Rule 1998 [online]. Available from URL: http://www.fda.gov/cder/pediatric [Accessed 2005 Aug 8]

3. Public law 107-109, Best Pharmaceuticals for children Act 2001 [online]. Available from URL: http://www.fda.gov/cder/pediatric [Accessed 2005 Aug 8]

4. Pediatric Research Equity Act of 2003 [online]. Available from URL: http://www.fda.gov/opacom/laws/prea.html [Accessed 2005 Aug 8]

5. Proposal for a Regulation of the Council and of the Parliament on Medicinal Products for Paediatric Use [online]. Available from URL: http://pharmacos.eudra.org/F2/Paediatrics/Index.htm [Accessed 2005 Jul 6]

6. Chalumeau M, Treluyer JM, Salanave B, et al. Off label and unlicensed drug use among French office based paediatricians. Arch Dis Child 2000; 83 (6): 502-5

7. Fontan JE, Combeau D, Brion F. Pediatric drug preparations in French hospitals: Pediatric Group of the French Clinical Pharmacy Society. Arch Pediatr 2000; 7 (8): $825-32$

8. Botting J. The history of thalidomide. Drug News Perspect 2002; 15 (9): 604-11

9. Conroy S, Choonara I, Impicciatore P, et al. Survey of unlicensed and off label drug use in paediatric wards in European countries. European Network for Drug Investigation in Children. BMJ 2000; 320 (7227): 79-82

10. Impicciatore $P$, Pandolfini $C$, Bonati M. Database could give children safer medicines [letter]. Nature 2000; 405 (6789): 882

11. Impicciatore $\mathrm{P}, \mathrm{Choonara} \mathrm{I}$, Clarkson $\mathrm{A}$, et al. Incidence of adverse drug reactions in paediatric in/out-patients: a systematic review and meta-analysis of prospective studies. Br J Clin Pharmacol 2001; 52 (1): 77-83

12. Lazarou J, Pomeranz BH, Corey PN. Incidence of adverse drug reactions in hospitalized patients: a meta-analysis of prospective studies. JAMA 1998; 279 (15): $1200-5$

13. Piotrovsky V. Population pharmacodynamic and pharmacokinetic modelling via mixed effects. Current Opin Drug Discov Devel 2000; 3 (3): 314-30

14. Sheiner L, Steimer JL. Pharmacokinetic/pharmacodynamic modelling in drug development. Annu Rev Pharmacol Toxicol 2000; 40: 67-95

15. Johnson T, Tanner M, Taylor C, et al. Enterocytic CYP3A4 in a paediatric population: developmental changes and the effects of celiac disease and cystic fibrosis. Br J Clin Pharmacol 2001; 51: 451-60

16. Johnson $\mathrm{T}$. The development of drug metabolizing enzymes and their influence on the susceptibility to adverse drug reactions in children. Toxicology 2003; 192: $37-48$ 
17. Reid AW, Anderson BJ, Futter ME. Relationship of muscle strength to potassium concentration in a hypokalaemic infant. Anaesth Intensive Care 1997; 25 (5): 525-7

18. Funck GA, Fischer M, Joos B. Quantification of in vivo replicative capacity of HIV-1 in different compartments of infected cells. J Acquir Immune Defic Syndr 2001; 26 (5): 397-404

19. Mandema J, Verrota D, Sheiner L. Pharmacometrics: building population pharmacokinetic-pharmacodynamic models: I. Models for covariate effects. J Pharmacokinet Biopharm 1992; 20 (5): 511-28

20. Sheiner L. Learning versus confirming in clinical drug development. Clin Pharmacol Ther 1997; 61 (3): 275-91

21. Tod M, Lokiec F, Bidault R, et al. Pharmacokinetics of oral acyclovir in neonates and in infants: a population analysis. Antimicrob Agents Chemother 2001; 45 (1): $150-7$

22. Holford N, Kimko H, Monteleone J, et al. Simulation of clinical trials. Annu Rev Pharmacol Toxicol 2000; 40: 209-34

23. Girard P, Cucherat M, Guez D. Simulation des essais cliniques dans le développement des médicaments. Therapie 2004; 59 (3): 287-304

24. Patoux A, Bleyzac N, Boddy A, et al. Comparison of nonlinear mixed-effect and non-parametric expectation maximisation modelling for Bayesian estimation of carboplatin clearance in children. Eur J Clin Pharmacol 2001; 57: 297-303

25. Rubie H, Doz F, Vassal G, et al. Individual dosing of carbolplatin based on drug monitoring in children receiving high-dose chemotherapy. Eur J Cancer 2003; 39: $1433-8$
26. Desfrere L, Zohar S, Morville P, et al. Dose-finding study of ibuprofen in patent ductus arteriosus using the continual reassessment method. J Clin Pharm Ther $2005 ; 30$ (2): 121-32

27. Gieschke R, Steimer JL. Pharmacometrics: modelling and simulation tools to improve decision making in clinical drug development. Eur J Drug Metab Pharmacokinet 2000; 25 (1): 49-58

28. Fabre E, Chevret S, Piechaud JF, et al. An approach for dose finding of drugs in infants: sedation by midazolam studied using the continual reassessment method. Br J Clin Pharmacol 1998; 46: 395-401

29. Guidance for industry: population pharmacokinetics, February 1999 [online]. Bethesda (MD): US Department of Health and Human Services, Food and Drug Administration, Center for Drug Evaluation and Research (CDER), Center for Biologics Evaluation and Research (CBER). Available from URL: http://www.fda.gov/cder/guidance/1852fnl.pdf [Accessed 2004 Dec 1]

30. Exposure-response relationships: study design, data analysis, and regulatory applications. 1998; 46: 395-401. Bethesda (MD): US Department of Health and Human Services, Food and Drug Administration, Center for Drug Evaluation and Research (CDER) Center for Biologics Evaluation and Research (CBER) April 2003 [online]. Available from URL: http://www.fda.gov/ohrms/dockets/ac/99/backgrd/3457b2a.pdf [Accessed 2004 Dec 1]

Correspondence and offprints: François Gueyffier, Centre d'Investigation Clinique, Hôpital Louis Pradel, 28 avenue du Doyen Lépine, 69500 Bron, France.

E-mail: francois.gueyffier@chu-lyon.fr 